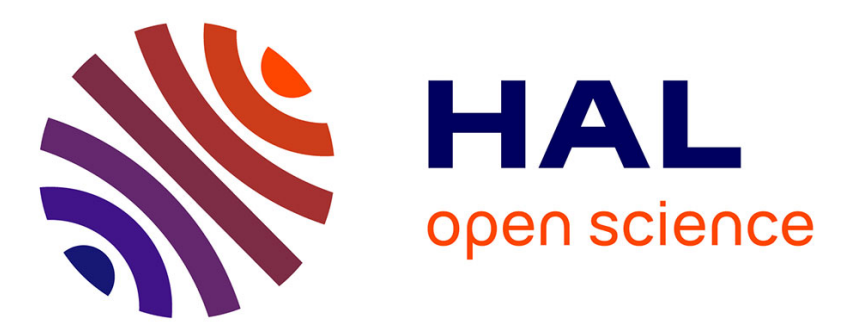

\title{
Symbolic numeric analysis of attractors in randomly generated piecewise affine models of gene networks
}

Etienne Farcot

\section{To cite this version:}

Etienne Farcot. Symbolic numeric analysis of attractors in randomly generated piecewise affine models of gene networks. International Conference on Symbolic and Algebraic Computation, 2006, Genoa, Italy. pp.79-86, 10.1145/1145768.1145787 . hal-00830070

\section{HAL Id: hal-00830070 https://hal.inria.fr/hal-00830070}

Submitted on 4 Jun 2013

HAL is a multi-disciplinary open access archive for the deposit and dissemination of scientific research documents, whether they are published or not. The documents may come from teaching and research institutions in France or abroad, or from public or private research centers.
L'archive ouverte pluridisciplinaire HAL, est destinée au dépôt et à la diffusion de documents scientifiques de niveau recherche, publiés ou non, émanant des établissements d'enseignement et de recherche français ou étrangers, des laboratoires publics ou privés. 


\title{
Symbolic Numeric Analysis of Attractors in Randomly Generated Piecewise Affine Models of Gene Networks
}

\author{
Etienne Farcot \\ INRIA - COMORE Project Team \\ 2004, route des Lucioles - BP 93 \\ 06902 Sophia Antipolis, France \\ etienne.farcot@sophia.inria.fr
}

\begin{abstract}
The goal of this paper is to present and experiment the computer aided analysis of phase portraits of some ordinary differential equations. The latter are piecewise affine, and have been primitively introduced as coarse-grained models of gene regulatory networks. Their simple formulation allows for numerical investigation, but their typical phase portrait is still largely unknown. They have been shown to present all the main aspects of nonlinear dynamics, including chaos. But it is still of interest to simulate random versions of these models, and to count and classify their attractors. This paper presents algorithms that allow for an automatic treatment of this kind, and apply it to four-dimensional sample systems. Contrary to previous studies, the latter have several thresholds in each direction, a fact whose consequences on the number and nature of attractors is discussed.

Categories and Subject Descriptors:

G.1.7 [Numerical Analysis]: Ordinary Differential Equations, I.6.1 [Simulation and Modeling]: Simulation Theory.

General Terms: Algorithms.

Keywords: Piecewise Affine Dynamical Systems, SymbolicNumeric Algorithms, Biological Networks.
\end{abstract}

\section{INTRODUCTION}

In this paper a special class of piecewise affine (PWA) differential equations is considered. Equations of this class may be written as :

$$
\frac{d x}{d t}=\Gamma(x)-\lambda x,
$$

where $x \in \mathcal{U} \subset \mathbb{R}_{+}^{n}, \lambda \in \mathbb{R}_{+}$and $\Gamma: \mathcal{U} \rightarrow \mathbb{R}_{+}^{n}$ is a piecewise constant map. As such, $\Gamma$ is not well defined on its whole domain $\mathcal{U}$. An alternative definition will be proposed later in this section. We denote its component functions $\left(\gamma_{1} \ldots \gamma_{n}\right)$. Since in practice each coordinate $x_{i}$ is bounded, it is relevant to set $\mathcal{U}=[0, \mathrm{M}]^{n}$ for some positive $\mathrm{M}$.

This kind of equations arise in theoretical biology, as a model of genetic and biochemical interaction networks. Their first

Permission to make digital or hard copies of all or part of this work for personal or classroom use is granted without fee provided that copies are not made or distributed for profit or commercial advantage and that copies bear this notice and the full citation on the first page. To copy otherwise, to republish, to post on servers or to redistribute to lists, requires prior specific permission and/or a fee.

ISSAC'06, July 9-12, 2006, Genova, Italy.

Copyright 2006 ACM 1-59593-276-3/06/0007 ...\$5.00. formulation was proposed by L. Glass in the seventies $[8,9$, 10]. These equations include some classical models of neural networks as a particular case $[7,17]$. In most of these cited works, each $\gamma_{i}$ is only allowed to take two distinct values, and is thus determined by a boolean function of $x$. We call binary systems this subclass of models. In general, $\gamma_{i}$ may take a finite number of distinct values, corresponding to rectangular regions in $\mathcal{U}$. Hence, (1) is strongly related to discrete-valued dynamical systems. In a biological context, those latter have been studied under a logical formalism [23], using the tools of qualitative dynamics [1], or as discrete dynamical systems over finite fields [16].

From a modelling viewpoint, each coordinate $x_{i}$ of vector $x$ represents a characteristic quantity of the $i$ th member in a finite population of $n$ interacting elements. For example, $x_{i}$ is the concentration of a protein whose production is induced from gene $i$, or $x_{i}$ is the voltage of a single neuron $i$. The positive coefficient $\lambda$ represents a degradation rate. In more general formulations, it should be written as a diagonal matrix. However, uniform decay rates are supposed in most works on systems of the form (1), for it greatly simplifies the analysis. This fact will appear in section 2 .

$\Gamma=\left(\gamma_{1} \ldots \gamma_{n}\right)$, on the other hand, describes coupling in the system. As it is piecewise constant, the domain of interest $\mathcal{U}$ is partitioned into rectangles of the form :

$$
B_{a}=B_{a_{1} \ldots a_{n}}=\prod_{i=1}^{n}\left[\theta_{i, a_{i}}, \theta_{i, a_{i}+1}\right],
$$

where the letter $B$ stands for 'box', since we will call such regions this way afterwards. This rectangular partition is biologically relevant. Actually, the processes involved here are submitted to rapid changes around some threshold values of the variables. More realistic models represent this by using sigmoidal functions, or so-called logoid functions, see [19], which tend to Heaviside step functions in the limit of infinite slope at threshold values. This limit correspond to piecewise constant production rates in rectangular regions of phase space. The thresholds are denoted

$$
\Theta_{i}=\left\{\theta_{i, j} \mid j=1 \ldots p_{i}\right\}
$$

in each direction $i$, where $p_{i} \in \mathbb{N}$ is the number of distinct qualitative states for the $i$ th variable. We suppose they are given in an ordered way : $\theta_{i, j}<\theta_{i, j+1}$. Although extreme values 0 and $M$ are not proper thresholds, we conventionally set $\theta_{i, 1}=0$ and $\theta_{i, p_{i}}=\mathrm{M}$, so that boxes actually form a partition of $\mathcal{U}$.

Since $\Gamma$ is constant on each box, which in turn is completely determined by its subscript $a$, it will be useful to consider $\Gamma$ 
as a map from the set of subscripts, $\mathcal{A}=\prod_{i=1}^{n} \mathbb{N}_{p_{i}}$ to $\mathbb{R}^{n}$. We denote $\mathbb{N}_{p_{i}}=\left\{1 \cdots p_{i}\right\}$. The set $\mathcal{A}$ will sometimes be called an alphabet in the sequel.

A discretizing mapping $\mathbf{d}: \bigcup_{a} \operatorname{int}\left(B_{a}\right) \rightarrow \mathcal{A}$ can be introduced here ; it associates to a point the subscripts of the box inside which it lies. It allows to relate the initial dynamical system (1) to a purely discrete one, with state space $\mathcal{A}$. This is a major tool in studying the dynamics of PWA systems like those we consider here. Observe that $\mathbf{d}$, as well as $\Gamma$, are not defined on the boundaries of boxes. This will be discussed in section 2.1.

Although these systems are conceived as simplifications of smooth nonlinear ones, they provide a wide variety of dynamical behaviours, which are still unsatisfactorily described. Images through $\mathbf{d}$ of the orbits of the initial system can be seen as infinite words on the alphabet $\mathcal{A}$. Among these words, the images of equilibrium points and periodic solutions of the continuous systems are periodic. However, some periodic words do not conversely correspond to periodic trajectories, but may be the images of stable foci, or even correspond to an empty set of trajectories. The problem of distinguishing which periodic words corresponds to limit cycles, and which correspond to foci, has been adressed early in the study of PWA systems like (1), by different authors $[11,12,18,22]$. All but the last reference concern the binary case, in which the only threshold in each direction is translated to zero, allowing a simplified analysis. In particular, trajectories on a given ray through the origin converge to the same limit. Hence, it is possible to project the phase portrait on a sphere $\mathcal{S}^{n-1}$ centered at the origin. Using such a projection, it has been proven that 3 dimensional binary systems cannot exhibit chaos [18], while some complicated periodic trajectories are proven to happen in a class of 4 dimensional systems [7].

More complex trajectories, represented by 'irrational' words on the alphabet $\mathcal{A}$, can be investigated using explicit computations. Actually, some recent studies [3, 5] improve known results about recurrent trajectories via analyzing a Poincaré return map, in the context of binary systems. This map is a fractional linear one, and an eigen-analysis of its linear term determines the existence and stability of its fixed points. Moreover, fixed points of this map shall only occur in a polyhedral cone that depends on the system's parameters. This cone corresponds to a forward invariant set of trajectories.

This paper's theoretical background is mainly an extension of those results on the return map and its fixed points, in the case of multiple thresholds. Those, along with preliminary working hypotheses and notations are provided in section 2. In section 3, the algorithms used to simulate, analyze, and randomly generate systems of the required form are described. Section 4 then presents results arising from an implementation of those algorithms, on a sample of systems with 4 variables. A discussion on the results obtained is provided in a concluding section.

\section{MATHEMATICAL BACKGROUND}

\subsection{The flow}

In this section, we recall the main definitions and properties that will be used afterwards. They are stated without proof, the latter having been established in a previous paper [6].

A system of form (1) induces a flow that can be formally written. In a given box $B_{a}, \Gamma$ is a constant vector, and we can easily compute the flow inside this box :

$$
\varphi^{a}(t, x) \doteq x(t)=f(a)+e^{-\lambda\left(t-t_{0}\right)}(x-f(a)) .
$$

Where $f(a)=\frac{1}{\lambda} \Gamma(a)$ is called focal point, because it is obviously attracting in the above equation. Hence, depending on its position with respect to $B_{a}$, it will be an asymptotically stable steady state, or the trajectory will encounter the boundary of the box. In the latter case, one assigns a new value to $\Gamma$ according to certain rules to be precised, and constructs a new leg of the trajectory by continuity. Observe that when $x$ is fixed and $t$ increases, the flow (3) defines a straight line. This is due to the uniformity of the decay rates: in a more general context, where different variables have distinct decay rates, trajectories are not straight line in boxes, complicating the whole analysis.

As mentioned in the previous section, $\Gamma$ is not defined on the boundaries of box. As $n$-rectangles, those boxes are polytopes, and thus we use the standard terminology about such objects. The uninformed reader is refered to [24]. The $k$-faces - i.e. faces of dimension $k$ - for $k \in\{0 \cdots n-1\}$ are in fact $k$-rectangles, which belong in general (i.e. except on the boundary of the whole domain) to $2^{n-k}$ adjacent $n$ dimensional boxes. Inside each of these boxes, the vector field is fixed. It follows that on all faces of codimension 2 or more, the flow is not well defined. On codimension 1 faces, on the other hand, some simple assumptions suffice to get a well defined continuous flow.

Assumption 1. $\forall a \in \mathcal{A}, f(a) \in \bigcup_{a \in \mathcal{A}} \operatorname{int}\left(B_{a}\right)$.

Assumption 2. $\forall i \in \mathbb{N}_{n}, \forall a, a^{\prime} \in \mathcal{A}, a-a^{\prime}= \pm \mathbf{e}_{i}$,

$$
\left(\mathbf{d}_{i}(f(a))-a_{i}\right)\left(\mathbf{d}_{i}\left(f\left(a^{\prime}\right)\right)-a_{i}^{\prime}\right)>0
$$

or

$$
\mathbf{d}_{i}(f(a))=a_{i} \text { and }\left(\mathbf{d}_{i}\left(f\left(a^{\prime}\right)\right)-a_{i}^{\prime}\right)\left(a_{i}-a_{i}^{\prime}\right)>0,
$$

or the same with a and $a^{\prime}$ exchanged.

Assumption 1 means that the focal points all lie inside the domain $\mathcal{U}$, and moreover, that none of them is on the boundary of a box. The first aspect implies that $\mathcal{U}=\bigcup_{a} B_{a}$ is positively invariant, and thus can be considered as the only region where relevant dynamics take place. The second one excludes a (rare) case which would otherwise cause technical complications without improving the model.

The second hypothesis concerns the case of autoregulation. For biological plausibility it should not be ignored, but it may lead to the use of generalized solutions in the sense of Filippov, i.e. differential inclusion, to have a mathematically rigorous definition of the flow [1, 14]. Assumption 2 precludes this kind of difficulties, by restricting the allowed dispositions of boxes and their focal points. Observe that two boxes $B_{a}$ and $B_{a^{\prime}}$ are adjacent in a single direction (i.e. through a facet) if and only if $\left\|a-a^{\prime}\right\|_{1}=1$, or equivalently if and only if there is some $i \in \mathbb{N}_{n}$ such that $a-a^{\prime}= \pm \mathbf{e}_{i}$ (where $\mathbf{e}_{i}$ is the $i$ th vector of the canonical basis). In words, the hypothesis means that the $i$ th component of the vector field does not change in sign when crossing a facet in 
direction $i$. Autoregulation (i.e. $\gamma_{i}(x)$ depends on $x_{i}$ ) is a necessary, but not sufficient condition for this configuration to happen. We thus do not reject all forms of autoregulation here. The situations avoided are schematically depicted in figure 1 .
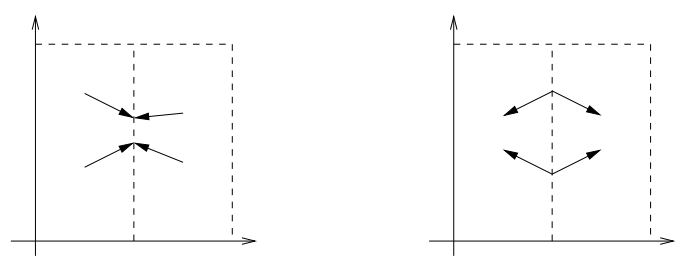

Figure 1: The two possible ambiguities due to autoregulation, often called black wall (on the left) and white wall (on the right).

\subsection{The transition map and its iterates}

Once the flow (3) is given in a box $B_{a}$, it is easy to compute the time and position at which it intersects the boundary of $B_{a}$, if ever. The possibility for each facet to be encountered by the flow uniquely depends on the position of the focal point : $\left\{x \mid x_{i}=\theta_{i, a_{i}}\right\}$ (resp. $\left\{x \mid x_{i}=\theta_{i, a_{i}+1}\right\}$ ) can be crossed if and only if $f_{i}<\theta_{i, a_{i}}$ (resp. $f_{i}>\theta_{i, a_{i}+1}$ ). According to this observation, we denote $I_{\text {out }}^{+}(a)=\{i \in$ $\left.\mathbb{N}_{n} \mid f_{i}>\theta_{i, a_{i}+1}\right\}$, and $I_{\text {out }}^{-}(a)=\left\{i \in \mathbb{N}_{n} \mid f_{i}<\theta_{i, a_{i}}\right\}$. Then, $I_{\text {out }}(a)=I_{\text {out }}^{+}(a) \cup I_{\text {out }}^{-}(a)$ is the set of escaping directions of $B_{a}$.

When it is unambiguous, we will omit the dependence on $a$, as we have already implicitely done with the focal point.

Now, in each direction $i \in I_{\text {out }}$ the time at which $\varphi(t, x)$ encounters the corresponding hyperplane, for $x \in B_{a}$, is given by:

$$
\tau_{i}(x)=\frac{-1}{\lambda} \ln \left(\min \left\{\frac{f_{i}-\theta_{i, a_{i}}}{f_{i}-x_{i}}, \frac{f_{i}-\theta_{i, a_{i}+1}}{f_{i}-x_{i}}\right\}\right) .
$$

Taking the minimum $\tau(x)=\min _{i \in I_{\text {out }}} \tau_{i}(x)$, and reinjecting it in equation (3), we get the exiting point of $B_{a}$ when starting at $x$. Since this process is intended to be repeated along trajectories, $x$ will generally lie on the boundary of the current box, except for the initial condition, which may however be chosen on a facet without loss of generality. We then get a transition map $\mathcal{T}^{a}: \partial B_{a} \rightarrow \partial B_{a}$ which can be explicited, omitting $a$ :

$$
\begin{aligned}
\mathcal{T} x & =\varphi(\tau(x), x) \\
& =f+\alpha(x)(x-f) .
\end{aligned}
$$

where $\alpha(x)=\exp (-\lambda \tau(x))$.

Observe that $\mathcal{T}$ can be geometrically interpreted : it is a central projection of center $f$, on a hyperplane supporting an exit facet. Hence, the image of a full set of points $S \subset$ $\partial B_{a}$ can be seen as the intersection of the polyhedral cone $f+\mathbb{R}_{+} S$, with exiting facets of $B_{a}$.

Now, the initial system (1) has been reduced to a discrete dynamical system, consisting in iterates of the above map $\mathcal{T}$ on a domain, denoted $\mathscr{D}$ : it is the union of all $n-1$ facets of boxes, taken without their boundary, nor these latters' finite-time preimages, as explained in section 2.1. The superscript $a$ will be systematically omitted afterwards, and $\mathcal{T}$ considered as a global map on $\mathscr{D}$. Actually, it can be proven [6] that under assumptions 1 and 2 there is always a single relevant $a$ such that $\mathcal{T} x=\mathcal{T}^{a} x$ for all $x \in \mathscr{D}$. Yet, we only care here with forward trajectories, since $\mathcal{T}^{-1}$ is not properly defined on the full domain $\mathscr{D}$.

Now, let $\mathbf{a}=a^{0} \ldots a^{k+1}$ a list of symbols in $\mathcal{A}$, such that the corresponding boxes are successively crossed by some flow line. The walls that are successively crossed by the flow are defined by: $W^{j}=\partial B_{a^{j}} \cap \partial B_{a^{j+1}}$, for all $j \in \mathbb{N}_{k} \cup\{0\}$.

Then, the following domain

$$
D_{\mathbf{a}} \doteq \bigcap_{j=0}^{k} \mathcal{T}^{-j}\left(W^{j}\right)
$$

defines all the initial conditions in $W^{0}$ such that the $k$ first iterates of $\mathcal{T}$ belong to the above defined walls.

Let also $f^{1} \ldots f^{k}$ be the focal points of the successive boxes, i.e. $f^{j}=f\left(a^{j}\right)$, and $s_{1}, \ldots, s_{k}$ the exit (or 'switching') directions in those boxes. Finally, let $\theta^{j}$ be the wall-defining threshold values : $\theta^{j} \in\left\{\theta_{s_{j}, a_{s_{j}}^{j}}, \theta_{s_{j}, a_{s_{j}}^{j}+1}\right\}$ is such that $W^{j} \subset$ $\left\{x \mid x_{s_{j}}=\theta^{j}\right\}$.

The $k$ th iterate of $\mathcal{T}$, for any $k \in \mathbb{N}$, can be expressed in the following form :

$$
\forall x \in D_{\mathbf{a}}, \quad \mathcal{T}^{k} x=f^{k}+\Delta_{s_{k}} \frac{F^{(k)}\left(x-f^{1}\right)}{\left\langle F^{(k)}\left(x-f^{1}\right), \mathbf{e}_{s_{k}}\right\rangle},
$$

where $\Delta_{s_{k}}=\theta^{k}-f_{s_{k}}^{k}$, and $F^{(k)} \in \mathbb{R}^{n \times n}$ is a matrix defined as the right to left product :

$$
\begin{aligned}
& F^{(k)}=\prod_{j=1}^{k-1}\left[\left(f^{j}-f^{j+1}\right) \mathbf{e}_{s_{j}}^{T}+\Delta_{s_{j}} I d\right] \\
& =\left[\left(f^{k-1}-f^{k}\right) \mathbf{e}_{s_{k-1}}^{T}+\Delta_{s_{k-1}} I d\right] \ldots \\
& \ldots\left[\left(f^{1}-f^{2}\right) \mathbf{e}_{s_{1}}^{T}+\Delta_{s_{1}} I d\right],
\end{aligned}
$$

for $k \geqslant 2$, and $F^{(1)}=I d$.

From this expression, it is possible to deduce some precise sufficient and necessary conditions for the existence of periodic orbits. A number of articles have dealt with such kind of conditions since early studies on Glass systems : chronologically $[11,12,18,22,3,6]$. We present here the main results in the form of a summarizing proposition, which is proven in [6]. First, it follows from (7) and (8) that the $\ell$-step map associated to a periodic sequence of walls, i.e. such that $a^{\ell}=a^{0}$ may be written as :

$$
\begin{aligned}
\mathcal{M}^{\ell} x-f^{1} & =\frac{\left[\left(f^{0}-f^{1}\right) \mathbf{e}_{s_{0}}^{T}+\Delta_{s_{0}} I d\right] F^{(\ell)}\left(x-f^{1}\right)}{\left\langle F^{(\ell)}\left(x-f^{1}\right), \mathbf{e}_{s_{0}}\right\rangle} \\
& =\frac{F^{(\ell+1)}\left(x-f^{1}\right)}{\left\langle F^{(\ell)}\left(x-f^{1}\right), \mathbf{e}_{s_{0}}\right\rangle} .
\end{aligned}
$$

Then we have the following two properties.

Proposition 1. Let $\mathbf{a}=a^{0} \ldots a^{\ell-1} a^{0}$ be the indices of a cyclic sequence of boxes in phase space with nonempty returning domain $D_{\mathbf{a}}$, and a return map written in the form (9). Assume the matrix $F^{(\ell+1)}$ has an eigenvector $v$ with real eigenvalue $\mu$. Then, the point :

$$
x^{*}=f^{1}+\frac{\mu}{\left\langle F^{(\ell)} v, \mathbf{e}_{s_{0}}\right\rangle} v
$$

is a fixed point of the return map, provided

$$
\frac{\mu}{\prod_{j=1}^{\ell} \Delta_{s_{j}}}>1, \quad \text { and } \quad x^{*} \in D_{\mathbf{a}} .
$$


Morevoer, it is asymptotically stable if, for any other eigenvalue $\eta$ of $F^{(\ell+1)}$ the following holds :

$$
|\mu|>|\eta| \text {. }
$$

If the inequality is weak, $x^{*}$ is stable, and it is unstable otherwise.

Proposition 2. For $\mathbf{a}=a^{0} \ldots a^{\ell}$, the set $D_{\mathbf{a}}$, as defined in equation (6) is properly described by the following list of inequalities, for each $j \in \mathbb{N}_{\ell-1}$ :

$$
\begin{aligned}
\forall i \in & I_{\text {out }}\left(a^{j}\right) \backslash\left\{s_{j}\right\}, \\
& \frac{\mathbf{e}_{i}^{T}}{\Delta_{i} \Delta_{s_{1}} \ldots \Delta_{s_{j}}}\left[\Delta_{s_{j}} I d-\Delta_{i} \mathbf{e}_{s_{j}}^{T}\right] F^{(j)}\left(x-f^{1}\right)>0,
\end{aligned}
$$

along with the $2(n-1)$ inequalities defining $W^{0} \supset D_{\mathbf{a}}$. The value $\theta^{i}$ being the escaping threshold in direction $i$ for the box $B_{a^{j}}, \Delta_{i}$ is similar to the already defined abbreviation, and equals $\theta^{i}-f_{i}^{j}$. Since for each $i, x-f^{1}$ is multiplied on the left by $a 1 \times n$ vector in inequality (10), the system of inequalities associated to each $j$ can be put in matrix form as $C^{(j)}\left(x-f^{1}\right)>0$, where the lines of $C^{(j)}$ are given by (10).

All these theoretical results are constructive, and may thus provide effective algorithms, which is the theme of the next section.

\section{ALGORITHMS}

First of all, the explicit expression of the return map and its iterates naturally leads to an algorithm for simulation of trajectories, given an initial condition. Then, asymptotically stable equilibria and limit cycles can be detected from simulation data. These two points form the topics of the first subsections, while a third one describes the algorithms we used to generate samples of random systems.

\subsection{Computation of trajectories}

The reduction of systems like (1) to a discrete time dynamical system yields an explicit scheme for numerical integration, as was first pointed out in [12]. Since that time, a number of papers have used this approach [2, 13, 15, 20]. We recall here the main steps of the algorithm.

The algorithm is defined for a fixed system of the form (1). Given an initial point $x^{0}$ and an integer nbiter, it computes a discretized orbit consisting in nbiter points. All of these lie on walls in phase space, excepted maybe $x^{0}$. The computed points are of course the nbiter first iterates of $\mathcal{T}$ on $x^{0}$. Due to the explicit form, eq. (3), of the flow in each box, it is important to note that any point on the continuous trajectory can be retrieved with arbitrary precision from the output of the simulation algorithm. This requires the knowledge of the sequence of boxes that are crossed by the computed flow line, as well as the times at which thresholds are met. We give a pseudo-code description in algorithm 1 . The symbol $\pm_{j}$ used for box updating, in this algorithm, is + (resp. - ) when the upper (resp. lower) threshold is met in direction $j$. Remind that $\arg \min _{\xi} F(\xi)$ is defined as the set $\{\xi \mid F(\xi)$ is minimal $\}$.

Several comments have to be made about this algorithm. First, the last step, at which a point of the trajectory is actually computed, uses the focal point of the box indexed by

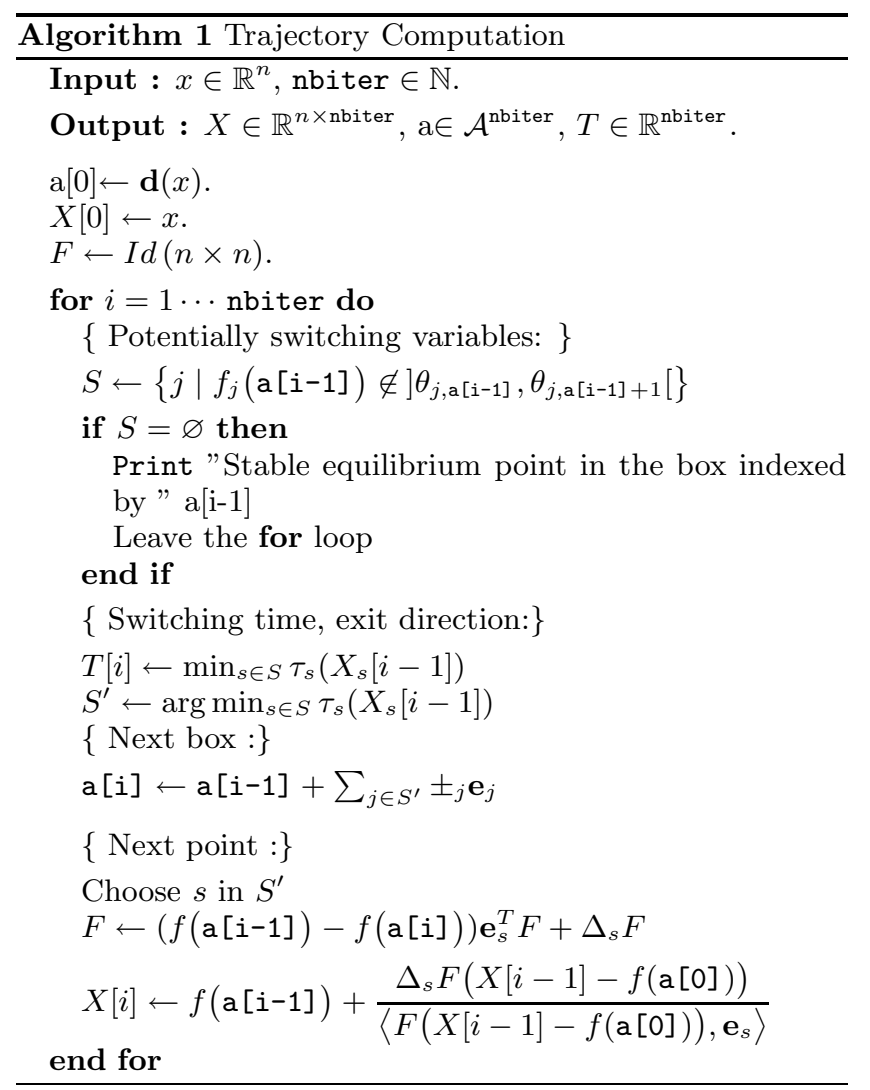

$a^{0}$, while it is $f^{1}=f\left(a^{1}\right)$ that is used in formula (7). This is due to the fact that the latter is valid for an initial condition $x \in W^{0}=B_{a^{0}} \cap B_{a^{1}}$, which is considered as a point in $B_{a^{1}}$ when applying $\mathcal{T}$.

Another remark is the fact that trajectories intersecting lower dimensional faces are considered as valid in the proposed algorithm, in contradiction with the discussion of section 2.2. This is due to the fact that while, in a theoretical context, $\mathcal{T}$ and all its iterates are well-defined - and even continuous - on $(n-1)$-faces of boxes, simulated trajectories may encounter lower dimensional faces. This is particularly true for binary systems studied in previous papers, for which thresholds are most often set to 0 and focal points taken randomly in the finite set $\{ \pm 1\}^{n}$. Such restrictions introduce symmetry, which lead to trajectories that often intersect low-dimensional faces. The most natural box update in such case is given in algorithm 1 : all $i$ such that $\tau_{i}$ is minimal are taken into account when updating box indices. Then, any $s \in I_{\text {out }}$ will provide the same transition map - up to the transition point $-\mathcal{T} x=\varphi(\tau(x), x)$, since $\tau_{s}(x)=\tau(x)$ for all such $s$. The image of such points, or equivalently the box to which they belong, is then ambiguous. Such situations appear rarely, but if ever our implementation updates all box subscripts simultaneously. All the trajectories presented in the rest of the paper are computed using this algorithm, which has been implemented in Matlab.

\subsection{Attractor analysis}

In algorithm 1, focal points lying in their corresponding box are automatically detected as asymptotically stable 

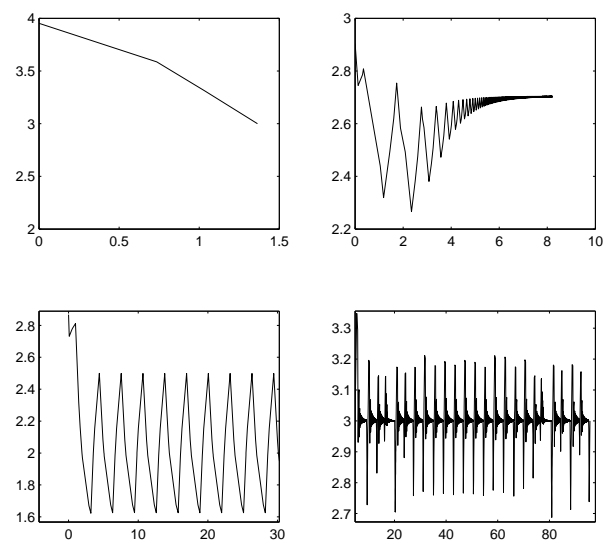

Figure 2: The four basic types of orbits, here taken from 4-dimensional examples. In each case, the first coordinate is plotted vs time. Upper-left corner: a node is detected and the algorithm stops. The actual orbit ends with a piece of exponential converging towards $f_{1}$. Upper-right corner: a stable focus. Lowerleft: a stable limit cycle. Lower-right: no periodic pattern is detected among 10000 steps of algorithm 1.

steady states. Such equilibria are usually called nodes. All attractors occuring in systems of the form (1) may be basically classified into four types, represented in figure 2 . The automatic detection and classification of attractors does only concern attractors of the last three types. It appears that the characterization of cycles and foci, as well as their stability, is just a direct application of propositions 1 and 2. Actually, they both concern periodic sequence of walls. They furthermore require the knowledge of matrices of the form $F^{(j)}$, of focal points $f^{j}$ and scaling coefficients $\Delta_{s_{j}}$, for intermediary $j$ in $\mathbb{N}_{\ell-1}$. These quantities are computed in the main loop, and thus can easily be added to the outputs of the algorithm 1.

Then, given a trajectory without node computed by this algorithm, the procedure used to detect and classify attractors consist in algorithm 2. The detection of stable foci is not specified in this algorithm, but will be discussed in section 4 . We discuss the nature of attractors that are unidentified by this procedure in section 4 .

\subsection{Generation of random networks}

Since all previous studies have been carried out in the context of binary systems, often with additional assumptions, we have to adapt the algorithms presented in the literature to generate random gene network models [13, 15, 20].

Similarly to those studies, we in fact generate systems with a fixed connectivity, denoted $K$. The latter is defined as the maximum number of variables that may influence a fixed other variable. The value of $K$ is often considered as being at most 2 or 3 in biological systems. Moreover, it is the main source of complexity when generating random discrete functions. Hence, it is more than usual to consider only low values of $K$. The decay rates is always fixed, and thus generating a random system amounts to generating a random

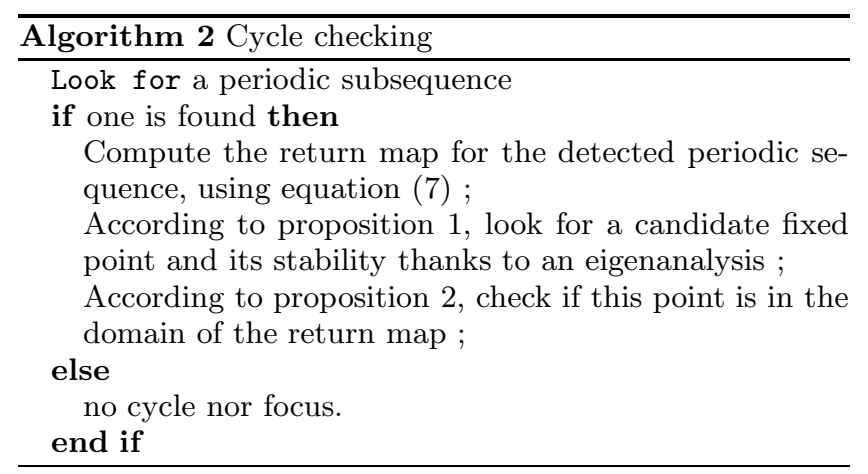

function $\Gamma$. In all the rest of the section, randomly generated quantities always follow a uniform repartition law. In concrete implementations, this is provided by pre-implemented routines, e.g. the RAND function in Matlab.

The principle of the generating algorithm, which appears in algorithm 3 is simple. For each $i \in \mathbb{N}_{n}$, the two following are randomly generated:

1) a subset $I(i) \subset \mathbb{N}_{n}$ of $K$ entries for the function $\gamma_{i}$.

2) a discrete function $\widetilde{\gamma}_{i}: \prod_{j \in I(i)} \mathbb{N}_{q_{j}} \rightarrow \mathbb{N}_{q_{i}}$.

Then, to each value of $\widetilde{\gamma}$, which belongs to $\mathcal{A}$, a random vector in $B_{\widetilde{\gamma}}$ is associated. All this requires the generation and storage of

$$
\mathscr{N}=n K+\sum_{i=1}^{n} \prod_{j \in I(i)} q_{j}
$$

floating numbers. Letting $q=\min _{i} q_{i}$ and $Q=\max _{i} q_{i}$ one gets $\mathscr{N} \in\left\{n\left(K+q^{K}\right) \ldots n\left(K+Q^{K}\right)\right\}$, and thus $\mathscr{N}$ is exponential in $K$. However, when $K \ll n$, it is linear in $n$. Essentially, the function $\widetilde{\gamma}$ is equivalent to a table with $K$ entries, each ranging in a finite domain. Such a structure is $K$-dimensional, and very bad suited for implementation. Thus we use a one-dimensional version of this map, i.e. a list. This is done via explicit bijections:

$$
\begin{aligned}
\Psi^{(i)}: & \prod_{j \in I(i)} \mathbb{N}_{q_{j}} \\
\left(a_{i_{1}} \ldots a_{i_{K}}\right) & \longmapsto \mathbb{N}_{q_{i_{1}} \ldots q_{i_{K}}} \\
& \longmapsto a_{i_{K}}+\sum_{j=1}^{K-1}\left(a_{i_{j}}-1\right) \prod_{m>j} q_{i_{m}},
\end{aligned}
$$

where $I(i)=\left\{i_{1} \ldots i_{K}\right\}$.

Observe that in the binary case, where $q_{i}=2$ and thus $\mathbb{N}_{q_{i}} \simeq\{0,1\}^{K}$, the above map is just the conversion from a binary expansion to the integer it represents.

Finally, the map $\Gamma$ is deduced from $\widetilde{\Gamma}$ in the most natural way: for each $a=\left(a_{1} \ldots a_{n}\right) \in \mathcal{A}$, a coordinate function $\gamma_{i}$ of $\Gamma$ only depends on the $a_{j}$ with $j \in I(i)$, and equals $\widetilde{\gamma}_{i}$ at those points.

Since $\widetilde{\Gamma}$ is randomly generated, it may happen that it does not depend effectively on all its input variables (for example, it may be the constant map). Hence, it is important to note that $K$ is not the actual connectivity of $\widetilde{\Gamma}$ (and thus $\Gamma$ ), but an upper-bound.

Note also that some restrictions have to be imposed on the values of the coordinate functions $\bar{\gamma}_{i}$, insofar as assumption 1 is required. Namely, they have to be outside the set $\lambda \Theta_{i}$, 


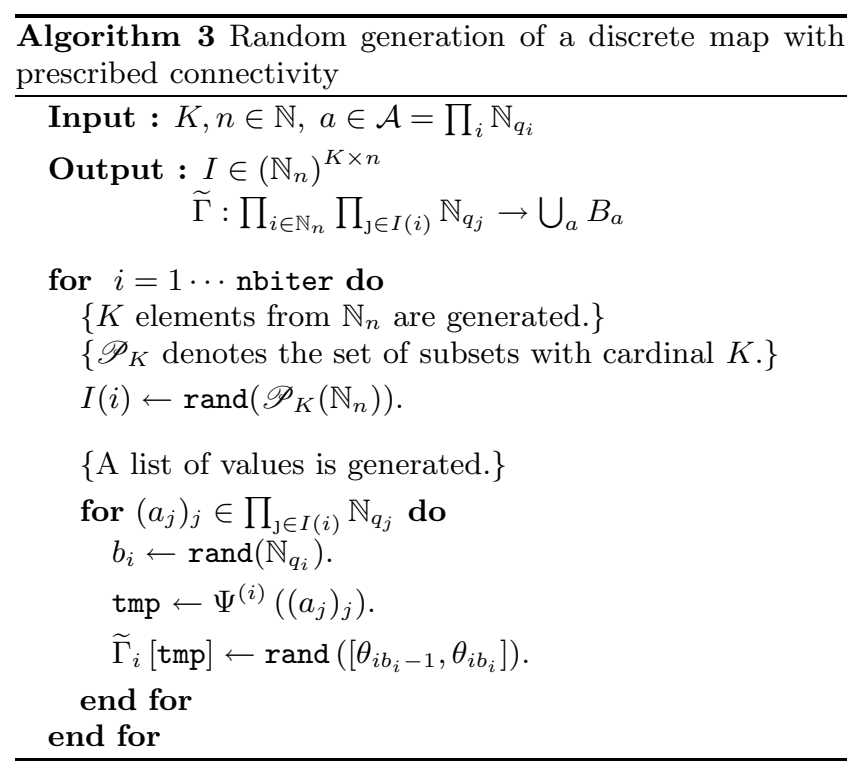

where $\Theta_{i}$ is the set of thresholds i direction $i$, equation (2). This does not appear in algorithm 3, but has been taken into account in the implementation.

Assumption 2, on the other hand, is easily satisfied, by requiring that, for all $i$, the set $I(i)$ does not contain $i$.

\section{RESULTS}

The algorithms presented in the previous section allow us to generate random systems of the form (1), compute trajectories of these systems, and then check the presence and nature of attractors in those trajectories. This general approach is not new, and has been used in previous studies [2, $13,15,20]$, always for binary systems. The main goal of all these studies is to obtain somme statistical insights in the nature, and proportion of each of the four types of attractors depicted on figure 2. The two major parameters whose influence on the latter are investigated are $n$ and $K$, the dimension of the systems, and their (maximal) connectivity. Here, we have chosen to consider a sample of non binary systems in $\mathbb{R}^{4}$, in order to gain some information on the influence of multiple thresholds in such systems. The main comparison will be [15], which provides the most similar study. We have generated systems with two intermediary thresholds - i.e. besides those defining the boundary of the whole domain - in each direction, and thus three discrete states in each direction. Such systems may thus be called ternary systems in $\mathbb{R}^{4}$ without autoregulation.

Nodes are detected directly from the computation of trajectories, algorithm 1. Cycles are analyzed from algorithm 2. Focus points can be guessed from the same algorithm. Actually, they correspond to fixed points of the return map along a cyclic sequence of walls, which belong to the boundary of a wall. Hence, when checking inequalities of proposition 2, they fail, but stand as limit cases, satisfying at least one equality of the form $x_{i}=\theta_{i, j}$. A further verification in our simulation was that the quantity $\mathcal{T}^{k+1} x-\mathcal{T}^{k} x$ tends toward 0 when $k$ increases, along an orbit $\left\{\mathcal{T}^{k} x\right\}$ through a periodic sequence of walls. Hence, only the fourth type of attractor remains unchecked.
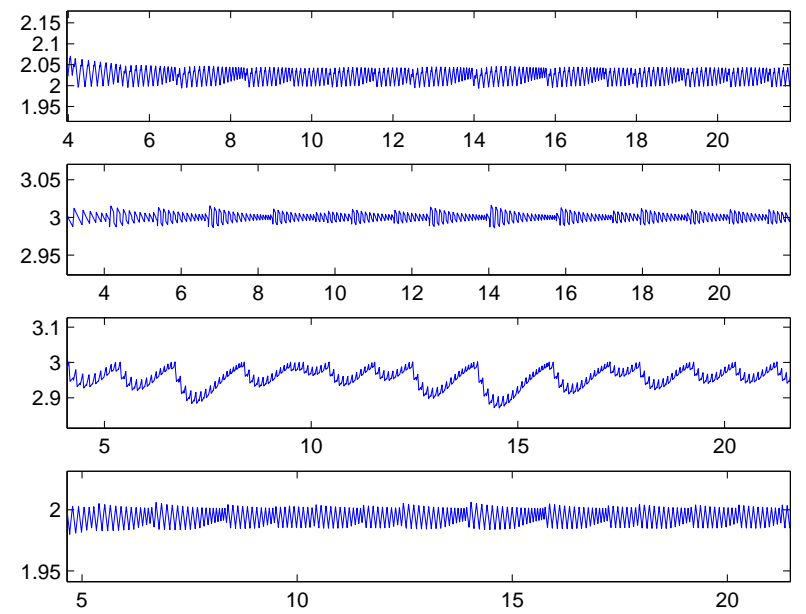

Figure 3: A recurrent orbit from a simulated system with four variables. No periodic sequence of walls detected in this portion of orbit.

The exact nature of such attractors is far from clear. They are often considered as chaotic orbits, but it is highly possible that they in fact are periodic orbits through very long sequences of walls, or transients of orbits whose asymptotic behaviour is simple. They may also be quasi-periodic ${ }^{1}$, which is hard to characterize numerically. However, chaos is proven to appear in such systems [3, 4, 21], and thus some unclassified attractors are certainly chaotic. A typical unclassified trajectory is represented in figure 3. Somehow abusively, we call chaotic the orbits that are of none of the three first types, in the following of this section.

Table (12) summarizes the results of our study, classified in terms of maximal connectivity. For each value of $K, 10000$ systems have been generated, using algorithm 3. For each system, a random initial condition has been chosen, and algorithm 1 has been applied for 1000 steps - or less if a node was detected. In the absence of node, the output of algorithm 1 was analyzed by algorithm 2 . If no cycle nor focus was detected, 4000 additional steps were computed in algorithm 1, and sent again to algorithm 2 - still in the absence of node. This provides :

\begin{tabular}{l|ccc}
$K$ & 1 & 2 & 3 \\
\hline \hline nodes & 8799 & 5728 & 4514 \\
foci & 949 & 2367 & 2336 \\
cycles & 873 & 2007 & 1996 \\
other & 100 & 253 & 260
\end{tabular}

This table should be compared with the following ;

\begin{tabular}{l|ccc}
$K$ & 1 & 2 & 3 \\
\hline \hline nodes & 1784 & 1455 & 1103 \\
foci & 171 & 518 & 876 \\
cycles & 43 & 27 & 19 \\
other & 2 & 0 & 2
\end{tabular}

\footnotetext{
${ }^{1}$ An orbit is called quasi-periodic if it is the sum of periodic
} orbits, whose periods are non-commensurable. 
taken from [15]. It corresponds to a sample of 2000 random networks for each value of $K$, all of which belong to the class of boolean systems ${ }^{2}$ without autoregulation.

The rank of the different types of attractors, classified only in terms of their number, is the same in both tables. The main difference is in their relative proportion, which varies for the last two types: our study provides a much higher proportion of cycles and unclassified trajectories. This fact can be attributed to several factors. Concerning unclassified trajectories, a notable shortcoming of the present study is the much lower number of iterations of algorithm 1. Actually, while we compute at most 5000 steps of this algorithm for each initial condition, the results from table (13) rely on as much as 30000 steps for the trajectories checked twice. Moreover, their numerical accuracy is set to $10^{-30}$, while we use the Matlab predefined precision $10^{-15}$. This suggests that a non-neglictible proportion of our unclassified trajectories correspond to transient behaviour, or worse, to numerical imprecisions, while most of those of table (13) actually represent chaotic-like orbits. However, figure 3 indicates that at least some of the trajectories we put in the fourth type are very likely, at least visually, to be chaotic. Another factor is the fact that ternary systems are more likely to present long transient behaviours, since they have a higher number of boxes in phase space.

The higher number of thresholds is also our main argument to explain the higher number of cycles in the present study, as opposed to those dealing with binary systems. Arguably, there are tow competing factors - besides the number of thresholds - in the explanation of this higher proportion of cycles. First, we only proceeded to relatively short integrations, and thus possibly missed some equilibria occuring after long transients. Second, our choice of random positions of focal points, differs from the choice of fixed symmetric points as in studies of binary systems. However, there is a geometric argument toward our claim. Actually, foci always appear at the intersection of several walls forming a periodic sequence under the action of $\mathcal{T}$. In non-binary systems, periodic sequences of walls may occur in such a way that at least two of these walls are disjoint. In such configurations, foci are impossible, and the only invariant sets provide periodic (or maybe chaotic) attractors. This intuitive fact is corroborated by examples like that of figure 4 , which are periodic cycles crossing a large number of boxes. Some of these boxes are crossed several times by this cycle, indicating its complicated shape in phase space. More importantly, this orbit crosses several thresholds in some directions, a fact that is evidently impossible in binary systems.

The important length of this cycle is not a new fact, since cycles crossing several hundreds of boxes have been detected in the study [2], about boolean systems.

Concerning the influence of connectivity, on the one hand it is qualitatively similar to the binary case in the sense that the number of nodes decreases when $K$ grows, while the number of foci increases. Its effect on other types of trajectories seems on the other hand opposite to the binary case. In terms of magnitude however, $K$ does not affect the number of cycles or chaotic orbits.

\footnotetext{
${ }^{2}$ Boolean systems are particular binary systems, in which the unique threshold is set to 0 , the degradation rate $\lambda=1$, and the production term $\Gamma$ - and thus the focal points have coordinates in $\{-1,+1\}$.
}
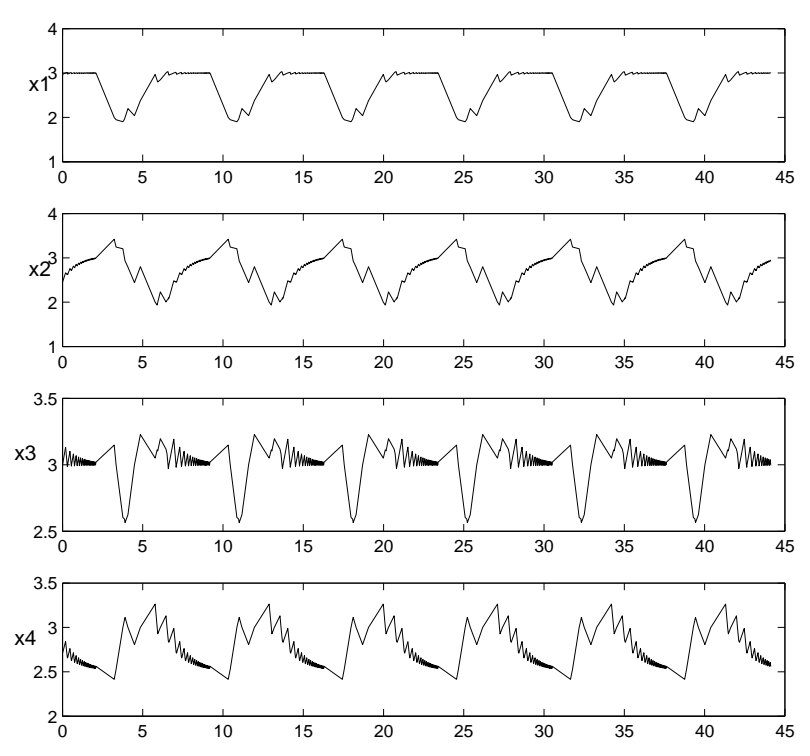

Figure 4: A stable periodic orbit from a ternary system in $\mathbb{R}^{4}$, taken among simulations summarized in table (12). Each coordinate is plotted vs time. This cycle crosses 94 sucessive boxes. thresholds are equal to 2 and 3 in each direction.

This leads to a final remark. As the reader may have observed, the total number of attractors in each column of table (12) differs from 10000. This is due to two reasons. For $K=3$, the total number of attractors is lower than 10000. The explanation comes from the numerical aspect of our implementation of algorithm 2. Actually, some matrices of the form $F^{(\ell+1)}$, see equation (7), were nearly singular in the generated data, leading to a trustless eigen-analysis. The orbits leading to such numerical difficulties were automatically rejected, explaining the small number of attractors for systems with connectivity 3 .

On the other hand, the number of attractors for systems with connectivity 1 and 2 exceeds 10000. This comes also from the actual implementation of algorithm 2, which computes all the eigenvectors of the matrix $F^{(\ell+1)}$, and then checks whether they lie in the returning domain, and if their eigenvalue satisfies the required inequality. It may happen that several eigenvectors satisfy the two conditions, in which case the return map admits several fixed points. One of them yields the attractor of the computed trajectory, while the others correspond to other orbits, and may in fact be unstable. A typical example of such situations arises when there is an unstable focus inside a stable limit cycle, a property that is ubiquitous in planar systems.

\section{CONCLUSION}

The numerical investigations presented in this paper only concern four-dimensional systems, and are thus merely a first inspection of systems of the form (1) with multiple thresholds. There are still a lot of open questions about such systems and their computer aided analysis. In this spirit a broader study, concerning more systems, with dif- 
ferent dimensions and connectivities, is a much appealing matter, and would have to be compared to previous studies on boolean systems, mainly [15].

From the computational point of view, the algorithm 2 consists in an eigenanalysis, matrix manipulations, and matrix inequalities checking. The algorithm 1, though of numeric nature, relies on an analytic formulation of the transition map. Hence, both algorithms should naturally lead to important improvements, thanks to a more symbolic oriented treatment. Notably, this would preclude the undesired rejection of trajectories discussed at the end of the last section. This may also concern the combination of several cycles, and some strange attractors, since such questions mostly involve tools from linear algebra [5].

In a more general perspective, the main contribution of this article is to show that the presence of multiple thresholds in piecewise affine models of gene networks has apparently the effect of increasing the number of periodic behaviours in those systems. Since multiple thresholds are known to occur in real-life systems, this indicates that biological networks may exhibit more oscillatory phenomena than expected from previous studies, which dealt with binary models. Also, it has to be kept in mind, that even with multiple thresholds, systems written as (1) are over-simplified models, and may only provide a schematic view of real biological phenomena. Mathematical analysis and computer experiments, are anyway a promising tool of investigation of this kind of biological systems, for which real biological experiments are still very difficult to set up today.

Acknowledgments. The author would like to thank two anonymous referees for their helpful and precise comments on this work.

\section{REFERENCES}

[1] H. de Jong, J.-L. Gouzé, C. Hernandez, M. Page, T. Sari, J. Geiselmann, Qualitative simulation of genetic regulatory networks using piecewise-linear models, Bull. Math. Biol., 66(2):301-340 (2004).

[2] R. Edwards, L. Glass, Combinatorial explosion in model gene networks, Chaos, 10(3):691-704 (2000).

[3] R. Edwards, Analysis of continuous-time switching networks, Physica D 146:165-199 (2000).

[4] R. Edwards, Chaos in neural and gene networks with hard switching, Diff. Eq. and Dyn. Sys., 9:187-220 (2001).

[5] R. Edwards, J.J. MacDonald, M.J. Tsatsomeros, On matrices with common invariant cones with applications in neural and gene networks, Linear Algebra Appl., 398:37-67 (2005).

[6] E. Farcot, Some geometric properties of piecewise affine biological network models, J. Math. Biol, 52(3):373-418 (2006).
[7] T. Gedeon, Attractors in continuous time switching networks, Commun. Pure Appl. Anal. (CPAA) vol. 2, No. 2, 187-209, (2003).

[8] L. Glass, S. Kauffman, The logical analysis of continuous non-linear biochemical control networks, J. Theor. Biol. 39:103-129 (1973).

[9] L. Glass, Classification of biological networks by their qualitative dynamics, J. Theor. Biol. 54:85-107 (1975).

[10] L. Glass, Combinatorial and topological methods in nonlinear chemical kinetics, J. Chem. Phys. 63:1325-1335 (1975).

[11] L. Glass, J.S. Pasternack, Prediction of limit cycles in mathematical models of biological oscillations, Bull. Math. Biol. 40:27-44 (1978).

[12] L. Glass, J.S. Pasternack, Stable oscillations in mathematical models of biological control systems, J. Math. Biol., 6:207-223 (1978).

[13] L. Glass, C. Hill, Ordered and disordered dynamics in random networks, Europhys. Lett. 41(6):599-604 (1998).

[14] J.L. Gouzé, T. Sari, A class of piecewise linear differential equations arising in biological models, Dynamical systems, 17:299-316 (2003).

[15] K. Kappler, R. Edwards, L. Glass, Dynamics in high-dimensional model gene networks, Signal Processing 83, 789-798 (2003).

[16] R. C. Laubenbacher, A computer algebra approach to biological systems, ISSAC 2003: 5-6 (2003).

[17] J.E. Lewis, L. Glass, Nonlinear and symbolic dynamics of neural networks, Neural Comput. 4:621-642 (1992).

[18] T. Mestl, E. Plahte, S.W. Omholt, Periodic solutions of piecewise-linear differential equations, Dyn. Stab. Syst., 10(2):179-193 (1995).

[19] T. Mestl, E. Plahte, S.W. Omholt, A mathematical framework for describing and analysing gene regulatory networks, J. Theor. Biol., 176:291-300 (1995).

[20] T. Mestl, R.J. Bagley, L. Glass, Common chaos in arbitrarily complex feedback networks, Phys. Rev. Lett. 79(4):653-656 (1997).

[21] T. Mestl, C. Lemay, L. Glass, Chaos in high-dimensional neural and gene networks, Physica D, 98:33-52 (1996).

[22] E.H. Snoussi, Qualitative dynamics of piecewise-linear differential equations: a discrete mapping approach, Dyn. Stab. Syst., 4(3-4):189-207 (1989).

[23] R. Thomas, Regulatory networks seen as asynchronous automata : a logical description, J. Theor. Biol., 153 (1991).

[24] G.M. Ziegler, Lectures on polytopes, Graduate Texts in Mathematics 152, Springer-Verlag, New York (1995). 